\title{
Adaptive Neural Output Feedback Control of Stochastic Nonlinear Systems with Unmodeled Dynamics
}

\author{
Xiaonan Xia and Tianping Zhang \\ Department of Automation, College of Information Engineering, Yangzhou University, Yangzhou 225127, China \\ Correspondence should be addressed to Tianping Zhang; tpzhang@yzu.edu.cn
}

Received 9 April 2014; Revised 17 June 2014; Accepted 4 July 2014

Academic Editor: Ming Gao

Copyright ( 2015 X. Xia and T. Zhang. This is an open access article distributed under the Creative Commons Attribution License, which permits unrestricted use, distribution, and reproduction in any medium, provided the original work is properly cited.

\begin{abstract}
An adaptive neural output feedback control scheme is investigated for a class of stochastic nonlinear systems with unmodeled dynamics and unmeasured states. The unmeasured states are estimated by K-filters, and unmodeled dynamics is dealt with by introducing a novel description based on Lyapunov function. The neural networks weight vector used to approximate the black box function is adjusted online. The unknown nonlinear system functions are handled together with some functions resulting from theoretical deduction, and such method effectively reduces the number of adaptive tuning parameters. Using dynamic surface control (DSC) technique, Itô formula, and Chebyshev's inequality, the designed controller can guarantee that all the signals in the closed-loop system are bounded in probability, and the error signals are semiglobally uniformly ultimately bounded in mean square or the sense of four-moment. Simulation results are provided to verify the effectiveness of the proposed approach.
\end{abstract}

\section{Introduction}

During the past decades, backstepping in [1] and dynamic surface control (DSC) in [2] have become two most popular methods for adaptive controller design. Many adaptive control schemes based on fuzzy/neural networks have been proposed for uncertain nonlinear systems using backstepping or dynamic surface control method in [3-13]. In the existing literature, three types of uncertainties were commonly considered, which included unknown system functions and parameter uncertainties and unmodeled dynamics. Unmodeled dynamics was dealt with by introducing an available dynamic signal in [3]. In addition, it was handled by a description method of Lyapunov function in [4]. In [4, 5], adaptive tracking control schemes were developed by backstepping and DSC for a class of strict-feedback uncertain nonlinear systems, respectively. In [7-10], adaptive control schemes were presented for a class of pure-feedback nonlinear systems. In [11-13], the adaptive tracking approaches for single-input single-output (SISO) nonlinear systems were extended to uncertain large-scale nonlinear systems.

When system states are assumed to be unmeasurable, output feedback adaptive control based on filters or observers has attracted much attention. In [14], K-filters were firstly proposed, and adaptive output feedback control was developed using K-filters. Inspired by the work in [14], robust adaptive output feedback control schemes were studied for SISO uncertain nonlinear systems in [15, 16]. In [17], combining backstepping technique with small-gain approach, indirect adaptive output feedback fuzzy control was developed. In [18], decentralized adaptive output-feedback control was designed based on high-gain K-filters and dynamic surface control method for a class of uncertain interconnected nonlinear systems.

It is well known that due to the stochastic terms and the extra quadratic variation terms resulting from the Itô differentiation rule, both the structures and the controller design of stochastic systems are commonly more complicated than those of deterministic systems. In the past decade, much effort has focused on the study of adaptive control schemes for uncertain stochastic nonlinear systems and the proof of the control system stability in probability sense. In [19-21], Deng et al. proposed the adaptive control scheme, based on backstepping for stochastic strict feedback or output-feedback nonlinear systems, and introduced a control Lyapunov function formula for stochastic disturbance 
attenuation earlier. In [22], by employing the stochastic Lyapunov-like theorem, adaptive backstepping state feedback control was developed for a class of stochastic nonlinear systems with unknown backlash-like hysteresis nonlinearities. In [23], the problem of decentralized adaptive outputfeedback control was discussed for a class of stochastic nonlinear interconnected systems. In $[24,25]$, output feedback adaptive fuzzy control approaches were considered using backstepping method for a class of uncertain stochastic nonlinear systems. In [26], by combining stochastic small-gain theorem with backstepping design technique, an adaptive output feedback control scheme was presented for a class of stochastic nonlinear systems with unmodeled dynamics and uncertain nonlinear functions. In [27], a concept of stochastic integral input-to-state stability (SiISS) using Lyapunov function was first introduced, and output feedback control was developed for stochastic nonlinear systems with stochastic inverse dynamics. In [28], two linear output feedback control schemes were studied to make the closed-loop system noiseto-state stable or globally asymptotically stable in probability. In [29], by using the homogeneous domination technique and appropriate Lyapunov functions, an output-feedback stabilizing controller was designed to be globally asymptotically stable in probability. In [30], the small-gain control method was investigated for stochastic nonlinear systems with SiISS inverse dynamics. In [31], based on a reduced-order observer, small-gain type condition on SiISS and stochastic LaSalle theorem, an output feedback controller was developed for stochastic nonlinear systems. In [32], an adaptive output feedback control scheme was investigated by combining Kfilters with DSC for a class of stochastic nonlinear systems with dynamic uncertainties and unmeasured states. In [33], adaptive control was developed using the backstepping method for a class of stochastic nonlinear systems with timevarying state delays and unmodeled dynamics.

Motivated by the above-mentioned results [4, 14, 32], in this paper, adaptive neural stochastic output feedback control is developed by combining K-filters with dynamic surface control to guarantee the stability of the closed-loop system. The main contributions of the paper lie in the following.

(i) Adaptive neural output feedback control is developed using K-filters and dynamic surface control for a class of stochastic nonlinear systems with unmodeled dynamics and unmeasured states. The advantage of the design is that once the local system constructed by the filter signals is stabilized, all the signals in the closed-loop system are bounded in probability.

(ii) Unmodeled dynamics is dealt with first by introducing a novel description based on Lyapunove function without using the dynamic signal to handle dynamic uncertainty in [32]. The novel description, which provides an effective method for dealing with unmodeled dynamics in output feedback adaptive controller design, is the development of original idea about handling unmodeled dynamics in [4].

(iii) Utilizing the boundedness of continuous function, the unknown nonlinear system functions are handled together with some functions produced in stability analysis, rather than directly approximated before stability analysis in $[6,8,9,11,12]$. Therefore the design effectively reduces the order of filters and the number of adjustable parameters of the whole system, without estimating $\Xi$ in [32].

(iv) Using bounded input bounded output (BIBO) stability and the filter special structure, the stability of the closed-loop system is proved. Therefore, the difficulty, that the transfer function cannot be used in a stochastic system while it was widely used to analyze the boundedness of the K-filters signals in the deterministic systems in $[4,14,16-18]$, is solved by the proposed stability analysis approach in this paper.

The rest of the paper is organized as follows. The problem formulation and preliminaries are given in Section 2. The neural filters are designed, and adaptive stochastic output feedback control is developed based on dynamic surface control method. The stability in the closed-loop system in probability sense is analyzed in Section 3. Simulation results are presented to illustrate the effectiveness of the proposed scheme in Section 4. Section 5 contains the conclusions.

\section{Problem Statement and Preliminaries}

Consider the following uncertain stochastic nonlinear systems with unmodeled dynamics:

$$
\begin{aligned}
& d z=q(z, y) d t \\
& d x_{1}=\left(x_{2}+f_{1}(y)+\Delta_{1}(z, y, t)\right) d t+g_{1}^{T}(y) d w \\
& d x_{\rho-1}=\left(x_{\rho}+f_{\rho-1}(y)+\Delta_{\rho-1}(z, y, t)\right) d t \\
& +g_{\rho-1}^{T}(y) d w \\
& d x_{\rho}=\left(x_{\rho+1}+f_{\rho}(y)+\Delta_{\rho}(z, y, t)+b_{m} \sigma(y) u\right) d t \\
& +g_{\rho}^{T}(y) d w \\
& d x_{n-1}=\left(x_{n}+f_{n-1}(y)+\Delta_{n-1}(z, y, t)+b_{1} \sigma(y) u\right) d t \\
& +g_{n-1}^{T}(y) d w \\
& d x_{n}=\left(f_{n}(y)+\Delta_{n}(z, y, t)+b_{0} \sigma(y) u\right) d t+g_{n}^{T}(y) d w \\
& y=x_{1},
\end{aligned}
$$

where $x=\left[x_{1}, x_{2}, \ldots, x_{n}\right]^{T} \in R^{n}$ is the state; $u \in R$ is the input, and $y \in R$ is the output; $\sigma(y) \neq 0$ is a known positive continuous function; $f_{i}(y)$ is the unknown smooth function; $z \in R^{n_{0}}$ is the unmodeled dynamics, and $\Delta_{i}(z, y, t)$ is the unknown smooth nonlinear dynamic disturbance; $b=$ $\left[b_{m}, \ldots, b_{1}, b_{0}\right]^{T} \in R^{m+1}, B(s)=b_{m} s^{m}+\cdots+b_{1} s+b_{0}$ is a Hurwitz polynomial; $\Delta_{i}(z, y, t)$ and $q(z, y)$ are the unknown Lipschitz 
functions; $w$ is an $r$-dimensional standard Brownian motion defined on the complete probability space $(\Omega, F, P)$ with $\Omega$ being a sample space, $F$ being a $\sigma$ field, and $P$ being a probability measure. In this paper, it is assumed that only output $y$ is available for measurement.

The control objective is to design output feedback adaptive control $u$ for system (1) such that the output $y$ follows the specified desired trajectory $y_{d}$, and all the signals of the closed-loop system are bounded in probability.

Assumption 1 (see [4]). The unknown nonlinear dynamic disturbances $\Delta_{i}(z, y, t), i=1,2, \ldots, n$, satisfy $\left|\Delta_{i}(z, y, t)\right| \leq$ $\rho_{i 1}(|y|)+\rho_{i 2}(y)\|z\|$, and $\rho_{i 1}(|y|)$ and $\rho_{i 2}(y)$ are the unknown nonnegative smooth functions, and $\|\cdot\|$ denotes the Euclidian norm of a vector.

Assumption 2. The system $\dot{z}=q(z, 0, t)-q(0,0, t)$ is globally exponentially stable when $z=0$; that is, there exists a Lyapunov function $W(t, z)$ satisfying

$$
\begin{gathered}
c_{1}\|z\|^{4} \leq W(z, t) \leq c_{2}\|z\|^{4} \\
\frac{\partial W}{\partial t}(z, t)+\frac{\partial W}{\partial z}(z, t)(q(z, 0, t)-q(0,0, t)) \\
\leq-c_{3}\|z\|^{4} \\
\left|\frac{\partial W}{\partial z}(z, t)\right| \leq c_{4}\|z\|^{3},
\end{gathered}
$$

where $c_{1}, c_{2}, c_{3}, c_{4}$ are positive constants, and there exists $c_{5} \geq 0$ such that $\|q(0,0, t)\| \leq c_{5}, \forall t \geq 0$.

Assumption 3. There exists an unknown function $\psi_{0}$, and $\psi_{0}(0)=0$, such that $\|q(z, y, t)-q(z, 0, t)\| \leq \psi_{0}(|y|)$ holds.

Assumption 4. The desired trajectory $x_{d}=\left[y_{d}, \dot{y}_{d}, \ddot{y}_{d}\right]^{T} \in \Omega_{d}$ is known, where $\Omega_{d}=\left\{x_{d}: y_{d}^{2}+\dot{y}_{d}^{2}+\ddot{y}_{d}^{2} \leq B_{0}\right\}$, and $B_{0}$ is a known constant.

Assumption 5. There exists a known constant $b_{\max }$ such that the following inequality $0<\left|b_{m}\right| \leq b_{\max }$ holds.

Remark 6. Assumption 2 is the extension of the description of unmodeled dynamics in [4], and it can effectively deal with unmodeled dynamics in output feedback adaptive controller design. To the best of authors' knowledge, this assumption is first addressed.

Consider the following stochastic nonlinear system:

$$
d x=f(t, x) d t+h^{T}(t, x) d w
$$

where $x \in R^{n}$ is the system state, $w$ is an $r$-dimensional standard Brownian motion, $f: R^{+} \times R^{n} \rightarrow R^{n}, h^{T}: R^{+} \times$ $R^{n} \rightarrow R^{n \times r}$ are locally Lipschitz and $f(t, 0), h(t, 0)$ are uniformly ultimately bounded. For any given $V(t, x(t)) \in C^{1,2}$, associated with the stochastic system (3), the infinitesimal generator $\ell$ is defined as follows:

$$
\begin{aligned}
\ell V(t, x(t))= & \frac{\partial V(t, x(t))}{\partial t}+\frac{\partial V(t, x(t))}{\partial x^{T}} f \\
& +\frac{1}{2} \operatorname{tr}\left\{h \frac{\partial^{2} V(t, x(t))}{\partial x^{T} \partial x} h^{T}\right\},
\end{aligned}
$$

where $\operatorname{tr}(A)$ is the trace of a matrix $A$.

Definition 7 (see [34]). The stochastic process $\{x(t)\}$ is said to be bounded in probability, if $\lim _{c \rightarrow \infty} \sup _{0 \leq t<\infty} P(|x(t)|>c)=$ 0 .

Definition 8. The solution $x(t)$ of system (3) is said to be semiglobally uniformly ultimately bounded (SGUUB) in $p$ th moment $(p \geq 1)$, if for some compact set $\Omega \subset R^{n}$ and any initial state $x_{0}=x\left(t_{0}\right) \in \Omega$, there exists a constant $\varepsilon>0$ and a time constant $T=T\left(\varepsilon, x_{0}\right)$ such that $E\left[\|x(t)\|^{p}\right] \leq \varepsilon$ for all $t>t_{0}+T$, especially, when $p=2$, it is usually called SGUUB in mean square.

Lemma 9 (see [32]). For any stochastic process $\{\xi(t)\}$, if there exists a positive integer $p$ and a positive constant $C_{0}$ such that $E|\xi(t)|^{p} \leq C_{0}, \forall t \geq 0$, then $\{\xi(t)\}$ is bounded in probability.

Lemma 10 (see [21]). Consider system (3) and suppose that there exists a $C^{2}$ function $V(t, x(t)): R^{n} \times R \rightarrow R^{+}$, two constants $c_{1}>0, c_{2} \geq 0$, class $\kappa_{\infty}$ functions $\mu_{1}, \mu_{2}$ such that

$$
\begin{aligned}
\mu_{1}(\|x\|) & \leq V(t, x) \leq \mu_{2}(\|x\|), \\
\ell V & \leq-c_{1} V+c_{2}
\end{aligned}
$$

for all $x \in R^{n}$ and $t>t_{0}$. Then, (i) for any initial state $x_{0} \in R^{n}$, there exists a unique strong solution $x(t)$ for system (3); (ii) the solution $x(t)$ of system (3) is bounded in probability;

(iii) $E\left[V\left(t_{0}, x\right)\right] \leq V\left(t_{0}, x_{0}\right) e^{-c_{1} t}+c_{2} / c_{1}, \forall t \geq t_{0}$.

In order to design filters and observer, (1) can be rewritten as follows:

$$
\begin{aligned}
\dot{z} & =q(z, y), \\
d x & =\left(A x+f(y)+F^{T}(y, u) b+\Delta\right) d t+g^{T}(y) d w, \\
y & =e_{1}^{T} x,
\end{aligned}
$$

where

$$
\begin{gathered}
A=\left[\begin{array}{cc}
0 & I_{n-1} \\
0 & 0
\end{array}\right], \\
f(y)=\left[\begin{array}{c}
f_{1}(y) \\
\vdots \\
f_{n}(y)
\end{array}\right],
\end{gathered}
$$




$$
\begin{aligned}
\Delta(z, y, t) & =\left[\begin{array}{c}
\Delta_{1}(z, y, t) \\
\vdots \\
\Delta_{n}(z, y, t)
\end{array}\right], \\
e_{1} & =[1,0, \ldots, 0]^{T}, \\
F^{T}(y, u) & =\left[\begin{array}{c}
0_{(\rho-1) \times(m+1)} \\
I_{m+1}
\end{array}\right] \sigma(y) u .
\end{aligned}
$$

\section{Adaptive Robust Controller Design and Stability Analysis}

3.1. Neural Filters and Controller Design. In order to estimate the state $x$, we introduce the following filters:

$$
\begin{aligned}
\dot{\xi} & =A_{0} \xi+L y, \quad \xi \in R^{n}, \\
\dot{\Omega}^{T} & =A_{0} \Omega^{T}+F^{T}(y, u), \quad \Omega^{T} \in R^{n \times(m+1)},
\end{aligned}
$$

where $A_{0}=A-L e_{1}^{T}, L=\left[l_{1}, \ldots, l_{n}\right]^{T}, A_{0}$ is a Hurwitz matrix; that is

$$
\begin{array}{r}
P A_{0}+A_{0}^{T} P=-h I, \\
P=P^{T}>0,
\end{array}
$$

where $h>0$ is a design constant.

Define the state estimate as follows:

$$
\widehat{x}=\xi+\Omega^{T} b
$$

The observer error is defined as $\varepsilon=x-\widehat{x}$. Thus

$$
\begin{aligned}
x & =\xi+\Omega^{T} b+\varepsilon, \\
d \varepsilon & =\left(A_{0} \varepsilon+f(y)+\Delta\right) d t+g^{T}(y) d w .
\end{aligned}
$$

Denote the columns of $\Omega^{T}$ as follows:

$$
\Omega^{T}=\left[v_{m}, \ldots, v_{1}, v_{0}\right], \quad \Omega^{T} \in R^{n \times(m+1)} .
$$

Inspired by the work in [14], the filters are designed as follows:

$$
\begin{aligned}
& \dot{\xi}=A_{0} \xi+L y, \quad \xi \in R^{n}, \\
& \dot{\lambda}=A_{0} \lambda+e_{n} \sigma(y) u, \quad \lambda \in R^{n}, \\
& \dot{v}_{j}=A_{0} v_{j}+e_{n-j} \sigma(y) u, \quad v_{j} \in R^{n}, j=0,1, \ldots, m .
\end{aligned}
$$

It is easy to show that

$$
\begin{aligned}
A_{0}^{j} e_{n} & =e_{n-j}, \quad j=0,1, \ldots, m, \\
v_{j} & =A_{0}^{j} \lambda, \quad j=0,1, \ldots, m,
\end{aligned}
$$

where $e_{i}$ denotes $n$ dimensional vector with the $i$ th element being one and other elements being all zeros, $i=1, \ldots, n$.
Let $v_{i, j}$ be the $j$ th element of the vector $v_{i}$ and $\lambda_{l}$ the $l$ th element of the vector $\lambda$, respectively. From [14], we know

$$
v_{i, j}=\left[\begin{array}{llll}
* & \cdots & * & 1
\end{array}\right]\left[\begin{array}{c}
\lambda_{1} \\
\lambda_{2} \\
\vdots \\
\lambda_{i+j}
\end{array}\right],
$$

$$
j=1, \ldots, \rho ; \quad i=0,1, \ldots, m ; \lambda_{l}=0, l>n .
$$

According to (11), we get

$$
\begin{aligned}
x_{2} & =\xi_{2}+\omega^{T} b+\varepsilon_{2}=\xi_{2}+\left[v_{m, 2}, \ldots, v_{1,2}, v_{0,2}\right] b+\varepsilon_{2} \\
& =b_{m} v_{m, 2}+\xi_{2}+\bar{\omega}^{T} \bar{b}+\varepsilon_{2},
\end{aligned}
$$

where $\omega^{T}$ denotes the second row of the matrix $\Omega^{T}, \xi_{2}$ denotes the second element of the vector $\xi$, and $\varepsilon_{2}$ is the second element of $\varepsilon$.

Substituting (18) into (1), it yields

$$
\begin{aligned}
& d y \\
& =\left(b_{m} v_{m, 2}+\xi_{2}+\bar{\omega}^{T} \bar{b}+\varepsilon_{2}+f_{1}(y)+\Delta_{1}(z, y, t)\right) d t \\
& \quad+g_{1}^{T}(y) d w,
\end{aligned}
$$

where

$$
\begin{aligned}
\omega^{T} & =\left[v_{m, 2}, v_{m-1,2}, \ldots, v_{1,2}, v_{0,2}\right], \\
\bar{\omega}^{T} & =\left[v_{m-1,2}, \ldots, v_{1,2}, v_{0,2}\right], \\
\bar{b}^{T} & =\left[b_{m-1}, \ldots, b_{1}, b_{0}\right] .
\end{aligned}
$$

In view of (19) and (14), the system used to design adaptive output feedback DSC in next section is addressed as follows:

$$
\begin{aligned}
d y= & \left(b_{m} v_{m, 2}+\xi_{2}+\bar{\omega}^{T} \bar{b}+\varepsilon_{2}+f_{1}(y)+\Delta_{1}\right) d t \\
& +g_{1}^{T}(y) d w, \\
\dot{v}_{m, i}= & v_{m, i+1}-l_{i} v_{m, 1}, \quad i=2, \ldots, \rho-1, \\
\dot{v}_{m, \rho}= & \sigma(y) u+v_{m, \rho+1}-l_{\rho} v_{m, 1} .
\end{aligned}
$$

3.2. Stochastic Adaptive Dynamic Surface Controller Design. In this subsection, according to (21) and by using dynamic surface control method, we propose an output feedback stochastic adaptive tracking control scheme. Similar to backstepping, the whole design needs $\rho$ steps.

For convenience, some notations are presented below. $\bar{s}_{i}=$ $\left[s_{1}, \ldots, s_{i}\right]^{T}, \bar{y}_{j}=\left[y_{2}, \ldots, y_{j}\right]^{T}$, where $s_{i}, y_{j}$ will be given in the controller design later, $i=1,2, \ldots, \rho, j=2, \ldots, \rho$. $y_{j}=\omega_{j}-\alpha_{j-1}, j=2, \ldots, \rho, \omega_{j}$ is the output of a firstorder filter with $\alpha_{i-1}$ as the input, and $\alpha_{i-1}$ is an intermediate control which will be developed for the corresponding $(i-$ 1)th subsystem. 
Define some Lyapunov functions as follows:

$$
\begin{aligned}
V_{\varepsilon} & =\varepsilon^{T} P \varepsilon \\
V_{W} & =\frac{1}{\lambda_{0}} W(z, t), \quad \lambda_{0}>0, \\
V_{s_{i}} & =\frac{1}{4} s_{i}^{4}, \\
V_{s W \varepsilon} & =V_{s_{1}}+V_{\varepsilon}+V_{W},
\end{aligned}
$$

where $W(z, t)$ is given in Assumption 2 .

Using Young's inequality, the infinitesimal generator of $V_{\varepsilon}$ satisfies

$$
\begin{aligned}
\ell V_{\varepsilon}= & \varepsilon^{T}\left(P A_{0}+A_{0}^{T} P\right) \varepsilon+2 \varepsilon^{T} P f(y)+2 \varepsilon^{T} P \Delta \\
& +\operatorname{tr}\left(g(y) P g^{T}(y)\right) \\
\leq & \varepsilon^{T}\left(P A_{0}+A_{0}^{T} P\right) \varepsilon+2 \varepsilon^{T} \varepsilon+\|P\|^{2}\|f(y)\|^{2} \\
& +\|P\|^{2}\|\Delta\|^{2}+\operatorname{tr}\left(g(y) P g^{T}(y)\right)
\end{aligned}
$$

According to Assumption 1 and by using Young's inequality, we obtain

$$
\begin{aligned}
\ell V_{\varepsilon} \leq & -(h-2) \varepsilon^{T} \varepsilon+\sum_{j=1}^{n}\|P\|^{2} f_{j}^{2}(y) \\
& +\sum_{j=1}^{n}\|P\|^{2}\left(\rho_{j 1}(|y|)+\rho_{j 2}(y)\|z\|\right)^{2} \\
& +\operatorname{tr}\left(g(y) P g^{T}(y)\right) \\
\leq & -(h-2) \varepsilon^{T} \varepsilon+\sum_{j=1}^{n}\|P\|^{2} f_{j}^{2}(y) \\
& +\sum_{j=1}^{n} 2\|P\|^{2}\left(\rho_{j 1}^{2}(|y|)+\rho_{j 2}^{2}(y)\|z\|^{2}\right) \\
& +\operatorname{tr}\left(g(y) P g^{T}(y)\right) \\
\leq & -(h-2) \varepsilon^{T} \varepsilon+\sum_{j=1}^{n}\|P\|^{2} f_{j}^{2}(y) \\
& +\operatorname{tr}\left(g(y) P g^{T}(y)\right) \\
& +\sum_{j=1}^{n} 2\|P\|^{2} \rho_{j 1}^{2}(|y|) \\
& \frac{16 n \lambda_{0}}{c_{3}} \sum_{j=1}^{n}\|P\|^{4} \rho_{j 2}^{4}(y)+\frac{c_{3}}{16 \lambda_{0}}\|z\|^{4} \\
& (h)
\end{aligned}
$$

Therefore, we have

$$
\begin{aligned}
\ell V_{s_{1}} & \\
= & s_{1}^{3}\left(b_{m} v_{m, 2}+\xi_{2}+\bar{\omega}^{T} \bar{b}+\varepsilon_{2}+f_{1}(y)+\Delta_{1}-\dot{y}_{d}\right) \\
& +\frac{3}{2} s_{1}^{2}\left\|g_{1}(y)\right\|^{2} .
\end{aligned}
$$

Choose the virtual control law $\alpha_{1}$ as follows:

$$
\alpha_{1}=\frac{\widehat{b}_{m}}{\widehat{b}_{m}^{2}+\beta}\left(-k_{1} s_{1}-\bar{\omega}^{T} \widehat{\bar{b}}-\xi_{2}-s_{1}^{3} \widehat{\theta}_{1}^{T} \psi_{1}(X)\right),
$$

where $\beta>0, k_{1}>0$ are design constants, $\widehat{\theta}_{1}, \widehat{b}_{m}, \widehat{\bar{b}}$ are the estimates of $\theta_{1}, b_{m}, \bar{b}$ at time $t$, respectively, and $\widetilde{b}_{m}=b_{m}-\widehat{b}_{m}$, $\widetilde{\theta}_{1}=\theta_{1}-\widehat{\theta}_{1}, \overline{\bar{b}}=\bar{b}-\widehat{\bar{b}}, \theta_{1}$ and $\psi_{1}(X)$ will be given later. Consider

$$
\begin{gathered}
\ell \alpha_{1}=\frac{\partial \alpha_{1}}{\partial y}\left(b_{m} v_{m, 2}+\xi_{2}+\bar{\omega}^{T} \bar{b}+\varepsilon_{2}+f_{1}(y)+\Delta_{1}(z, y, t)\right) \\
+\frac{\partial \alpha_{1}}{\partial \widehat{b}_{m}} \dot{\vec{b}}_{m}+\frac{\partial \alpha_{1}}{\partial \xi^{T}} \dot{\xi}+\frac{\partial \alpha_{1}}{\partial \widehat{\theta}_{1}^{T}} \dot{\hat{\theta}}_{1}+\frac{\partial \alpha_{1}}{\partial y_{d}} \dot{y}_{d} \\
+\frac{\partial \alpha_{1}}{\partial \dot{y}_{d}} \ddot{y}_{d}+\frac{1}{2} \frac{\partial^{2} \alpha_{1}}{\partial y^{2}} g_{1}^{T}(y) g_{1}(y), \\
d \alpha_{1}=\ell \alpha_{1} d t+\frac{\partial \alpha_{1}}{\partial y} g_{1}^{T}(y) d w .
\end{gathered}
$$

A first-order filter with $\alpha_{1}$ as the input is designed as follows:

$$
\tau_{2} \dot{\omega}_{2}+\omega_{2}=\alpha_{1}, \quad \omega_{2}(0)=\alpha_{1}(0) \text {. }
$$


Let $y_{2}=\omega_{2}-\alpha_{1}$; thus, $\dot{\omega}_{2}=-y_{2} / \tau_{2}$. Since $v_{m, 2}=s_{2}+y_{2}+\alpha_{1}$, using Young's inequality, it yields

$$
\begin{aligned}
\ell V_{s_{1}} \leq & -\left(k_{1}-\frac{3}{2} b_{\max }\right) s_{1}^{4}+\widetilde{b}_{m} s_{1}^{3} \alpha_{1}+\frac{b_{\max }}{4} s_{2}^{4} \\
& +\frac{b_{\max }}{4} y_{2}^{4}+s_{1}^{3} \bar{\omega}^{T} \tilde{\bar{b}}-s_{1}^{3} \tilde{\theta}_{1}^{T} \psi_{1}(X) \\
& -\frac{s_{1}^{3} \beta}{\widehat{b}_{m}^{2}+\beta}\left(-k_{1} s_{1}-\bar{\omega}^{T} \hat{\bar{b}}-\xi_{2}-s_{1}^{3} \widehat{\theta}_{1}^{T} \psi_{1}(X)\right) \\
& +s_{1}^{3} \varepsilon_{2}+s_{1}^{3} f_{1}(y)+s_{1}^{3} \Delta_{1}-s_{1}^{3} \dot{y}_{d} \\
& +\frac{3}{2} s_{1}^{2}\left\|g_{1}(y)\right\|^{2} .
\end{aligned}
$$

From Assumption 1, we obtain

$$
\begin{aligned}
\left|s_{1}^{3}\right|\left|\Delta_{1}\right| \leq & \left|s_{1}^{3}\right| \rho_{11}(|y|)+\left|s_{1}^{3}\right| \rho_{12}(y)\|z\| \\
\leq & \frac{3}{4} s_{1}^{4}+\frac{1}{4} \rho_{11}^{4}(|y|)+\frac{3}{2} \sqrt[3]{\frac{\lambda_{0}}{2 c_{3}}} s_{1}^{4} \rho_{12}^{4 / 3}(y) \\
& +\frac{c_{3}}{16 \lambda_{0}}\|z\|^{4} .
\end{aligned}
$$

In view of (24), (25), (32), and (33) and by using Young's inequality, we obtain

$$
\begin{aligned}
\ell V_{s W \varepsilon} \leq & -(h-2) \varepsilon^{T} \varepsilon-\left(k_{1}-\frac{3}{2} b_{\max }-\frac{3}{4}\right) s_{1}^{4} \\
& -\frac{c_{3}}{2 \lambda_{0}}\|z\|^{4}+\widetilde{b}_{m} s_{1}^{3} \alpha_{1}+s_{1}^{3} \bar{\omega}^{T} \widetilde{\bar{b}}+\frac{b_{\max }}{4} s_{2}^{4} \\
& +\frac{b_{\max }}{4} y_{2}^{4}+\left|s_{1}^{3}\right| S+s_{1}^{3} H_{1}(X)+Q(y)+\frac{1}{4} \varepsilon_{2}^{2} \\
& -s_{1}^{3} \tilde{\theta}_{1}^{T} \psi_{1}(X)+\frac{16 c_{4}^{4} c_{5}^{4}}{\lambda_{0} c_{3}^{3}}+1,
\end{aligned}
$$

where

$$
\begin{gathered}
Q(y)=\sum_{j=1}^{n}\|P\|^{2} f_{j}^{2}(y)+\sum_{j=1}^{n} 2\|P\|^{2} \rho_{j 1}^{2}(|y|) \\
+\frac{16 n \lambda_{0}}{c_{3}} \sum_{j=1}^{n}\|P\|^{4} \rho_{j 2}^{4}(y)+\frac{16 c_{4}^{4}}{\lambda_{0} c_{3}^{3}} \psi_{0}^{4}(|y|) \\
+\frac{1}{4} \rho_{11}^{4}(|y|)+\operatorname{tr}\left(g(y) P g^{T}(y)\right), \\
H_{1}(X)=\frac{3}{2} \sqrt[3]{\frac{\lambda_{0}}{2 c_{3}}} s_{1} \rho_{12}^{4 / 3}(y)-\dot{y}_{d}+\frac{9}{16} s_{1}\left\|g_{1}(y)\right\|^{4}+s_{1}^{3}, \\
X=\left[s_{1}, y_{d}, \dot{y}_{d}\right]^{T} \in R^{3} .
\end{gathered}
$$

$S\left(s_{1}, \widehat{b}_{m}, \widehat{\bar{b}}, \widehat{\theta}_{1}, \xi, \bar{\lambda}_{m+2}, y_{d}\right)$ is a nonnegative continuous function, and

$$
\begin{aligned}
& \left|f_{1}(y)-\frac{\beta}{\hat{b}_{m}^{2}+\beta}\left(-k_{1} s_{1}-\bar{\omega}^{T} \overline{\bar{b}}-\xi_{2}-s_{1}^{3} \widehat{\theta}_{1}^{T} \psi_{1}(x)\right)\right| \\
& \quad \leq S\left(s_{1}, \widehat{b}_{m}, \hat{\bar{b}}, \widehat{\theta}_{1}, \xi, \bar{\lambda}_{m+2}, y_{d}\right),
\end{aligned}
$$

where $\bar{\lambda}_{m+2}=\left[\lambda_{1}, \ldots, \lambda_{m+2}\right]^{T}$.

Let $\Omega_{X}=\left\{X \mid\|X\| \leq M_{X}\right\} \subset R^{3}$ be a given compact set with $M_{X}>0$ being a design constant, and let $\theta_{1}^{T} \phi_{1}(X)$ be the approximation of the radial basis function neural networks on the compact set $\Omega_{X}$ to $H_{1}(X)$. Then, we have $H_{1}(X)=$ $\theta_{1}^{T} \psi_{1}(X)+B_{1}(X)$, where $B_{1}(X)$ denotes the approximation error and $\psi_{1}(X)=\left[\psi_{11}(X), \ldots, \psi_{1 M_{1}}(X)\right]^{T} \in R^{M_{1}}$ denotes the basis function vector with $\psi_{1 j}(X)$ being chosen as the commonly used Gaussian functions, which have the form

$$
\psi_{1 j}(X)=\exp \left[-\frac{\left\|X-\mu_{1 j}\right\|^{2}}{b_{1 j}^{2}}\right]
$$

$j=1, \ldots, M_{1}$, and $\mu_{1 j}$ is the center of the receptive field and $b_{1 j}$ is the width of the Gaussian function; $\theta_{1}$ is an adjustable parameter vector.

According to (34) and by using Young's inequality, it yields

$$
\begin{aligned}
\ell V_{s W \varepsilon} \leq & -\left(h-\frac{9}{4}\right) \varepsilon^{T} \varepsilon-\left(k_{1}-\frac{3}{2} b_{\max }-\frac{3}{2}\right) s_{1}^{4}-\frac{c_{3}}{2 \lambda_{0}}\|z\|^{4} \\
& +\widetilde{b}_{m} s_{1}^{3} \alpha_{1}+s_{1}^{3} \bar{\omega}^{T} \widetilde{\bar{b}}+\frac{b_{\max }}{4} s_{2}^{4}+\frac{b_{\max }}{4} y_{2}^{4} \\
& +\frac{1}{4} S^{4}+s_{1}^{3}\left(\theta_{1}^{T} \psi_{1}(X)+B_{1}(X)\right)+Q(y) \\
& -s_{1}^{3} \widetilde{\theta}_{1}^{T} \psi_{1}(X)+\frac{16 c_{4}^{4} c_{5}^{4}}{\lambda_{0} c_{3}^{3}}+1 .
\end{aligned}
$$

There exists a nonnegative continuous function $\kappa\left(s_{1}, y_{d}, \dot{y}_{d}\right)$ satisfying

$$
\left|B_{1}(X)\right| \leq \kappa\left(s_{1}, y_{d}, \dot{y}_{d}\right) .
$$

Using Young's inequality, we have

$$
\begin{aligned}
\ell V_{s W \varepsilon} \leq & -\left(h-\frac{9}{4}\right) \varepsilon^{T} \varepsilon-\left(k_{1}-\frac{3}{2} b_{\max }-\frac{9}{4}\right) s_{1}^{4} \\
& -\frac{c_{3}}{2 \lambda_{0}}\|z\|^{4}+\widetilde{b}_{m} s_{1}^{3} \alpha_{1}+s_{1}^{3} \bar{\omega}^{T} \tilde{\bar{b}}+\frac{b_{\max }}{4} s_{2}^{4} \\
& +\frac{b_{\max }}{4} y_{2}^{4}+\frac{1}{4} S^{4}+Q(y)+s_{1}^{3} \widetilde{\theta}_{1}^{T} \psi_{1}(X) \\
& +\frac{1}{4} \kappa^{4}+C_{0},
\end{aligned}
$$

where $C_{0}=16 c_{4}^{4} c_{5}^{4} / \lambda_{0} c_{3}^{3}+1$. 
Step $i(2 \leq i \leq \rho-1)$. Define the $i$ th dynamic surface $s_{i}=$ $v_{m, i}-\omega_{i}$, thus

$$
\dot{s}_{i}=v_{m, i+1}-l_{i} v_{m, 1}-\dot{\omega}_{i}
$$

Select the virtual control law $\alpha_{i}$ as follows:

$$
\begin{aligned}
\alpha_{i} & =-k_{i} s_{i}+l_{i} v_{m, 1}+\dot{\omega}_{i}, \\
\ell \alpha_{i} & =-k_{i}\left(v_{m, i+1}-l_{i} v_{m, 1}-\dot{w}_{i}\right)+l_{i} \dot{v}_{m, 1}-\frac{\ell y_{i}}{\tau_{i}}, \\
d \alpha_{i} & =\ell \alpha_{i} d t+\frac{\partial \alpha_{i}}{\partial y} g_{1}^{T}(y) d w .
\end{aligned}
$$

A first-order filter with the input $\alpha_{i}$ is designed as follows:

$$
\tau_{i+1} \dot{\omega}_{i+1}+\omega_{i+1}=\alpha_{i}, \quad \omega_{i+1}(0)=\alpha_{i}(0),
$$

where $\tau_{i+1}>0$ is a design constant.

Let $y_{i+1}=\omega_{i+1}-\alpha_{i}$. Then $\dot{\omega}_{i+1}=-y_{i+1} / \tau_{i+1}$. Noting $v_{m, i+1}=s_{i+1}+y_{i+1}+\alpha_{i}$, in view of (41) and (42), we obtain

$$
\begin{aligned}
\ell V_{s_{i}} & =s_{i}^{3} \dot{s}_{i}=s_{i}^{3}\left(v_{m, i+1}-l_{i} v_{m, 1}-\dot{w}_{i}\right) \\
& =-k_{i} s_{i}^{4}+s_{i}^{3} s_{i+1}+s_{i}^{3} y_{i+1} \\
& \leq-\left(k_{i}-\frac{3}{2}\right) s_{i}^{4}+\frac{1}{4} s_{i+1}^{4}+\frac{1}{4} y_{i+1}^{4} .
\end{aligned}
$$

Step $\rho$. The control law will be determined in this step. Define the $\rho$ th dynamic surface as $s_{\rho}=v_{m, \rho}-\omega_{\rho}$. The derivative of $s_{\rho}$ is

$$
\dot{s}_{\rho}=\sigma(y) u+v_{m, \rho+1}-l_{\rho} v_{m, 1}-\dot{\omega}_{\rho} .
$$

Choose the control law as follows:

$$
u=\frac{\left(-k_{\rho} s_{\rho}-v_{m, \rho+1}+l_{\rho} v_{m, 1}+\dot{\omega}_{\rho}\right)}{\sigma(y)} .
$$

In view of (45) and (46), we have

$$
\ell V_{s_{\rho}}=s_{\rho}^{3} \dot{s}_{\rho}=-k_{\rho} s_{\rho}^{4} .
$$

The parameters $\widehat{\theta}_{1}, \widehat{b}_{m}$, and $\widehat{\bar{b}}$ are updated as follows:

$$
\begin{aligned}
& \dot{\hat{\theta}}_{1}=\gamma_{1}\left(s_{1}^{3} \psi_{1}(X)-\sigma_{1} \widehat{\theta}_{1}\right), \\
& \dot{\hat{b}}_{m}=\gamma_{2}\left(s_{1}^{3} \alpha_{1}-\sigma_{2} \widehat{b}_{m}\right), \\
& \dot{\overline{\vec{b}}}=\gamma_{3}\left(s_{1}^{3} \bar{\omega}-\sigma_{3} \hat{\bar{b}}\right),
\end{aligned}
$$

where $\gamma_{1}, \gamma_{2}, \gamma_{3}, \sigma_{1}, \sigma_{2}, \sigma_{3}$ are the design constants.
3.3. Stability Analysis of Adaptive Control System. In this subsection, we will discuss the stability analysis of the closedloop system. Firstly we define some Lyapunov functions and compact sets as follows:

$$
\begin{aligned}
V_{1}= & \frac{1}{2} s_{1}^{4}+2 V_{\varepsilon}+\frac{1}{\gamma_{1}} \widetilde{\theta}_{1}^{T} \widetilde{\theta}_{1}+\frac{1}{\gamma_{2}} \widetilde{b}_{m}^{2}+\frac{1}{\gamma_{3}} \widetilde{\bar{b}}^{T} \widetilde{\bar{b}} \\
& +\frac{c_{3}}{\lambda_{0}}\|z\|^{4}, \\
V_{i}= & \sum_{j=1}^{i} \frac{1}{2} s_{j}^{4}+2 V_{\varepsilon}+\sum_{j=2}^{i} \frac{1}{2} y_{j}^{4}+\frac{1}{\gamma_{1}} \widetilde{\theta}_{1}^{T} \widetilde{\theta}_{1}+\frac{1}{\gamma_{2}} \widetilde{b}_{m}^{2} \\
& +\frac{1}{\gamma_{3}} \widetilde{\bar{b}}^{T} \widetilde{\bar{b}}+\frac{c_{3}}{\lambda_{0}}\|z\|^{4}, \quad i=2, \ldots, \rho, \\
\Omega_{1}= & \left\{\left(s_{1}, \varepsilon, \widehat{\theta}_{1}, \widehat{b}_{m}, \widehat{\bar{b}},\|z\|\right): V_{1} \leq p\right\} \subset R^{p_{1}}, \\
\Omega_{i}= & \left\{\left(\bar{s}_{i}, \bar{y}_{i}, \varepsilon, \widehat{\theta}_{1}, \widehat{b_{m}}, \widehat{\bar{b}},\|z\|\right): V_{i} \leq p\right\} \subset R^{p_{i},}
\end{aligned}
$$

where $i=2, \ldots, \rho, p>0$ is a design constant, $p_{i}=2 i+M_{1}+$ $n+m+1$. It is easy to know that $\Omega_{1} \times R^{p_{\rho}-p_{1}} \supset \Omega_{2} \times R^{p_{\rho}-p_{2}} \supset$ $\cdots \supset \Omega_{\rho-1} \times R^{p_{\rho}-p_{\rho-1}} \supset \Omega_{\rho}$.

According to $y_{2}=\omega_{2}-\alpha_{1}$, we obtain

$$
\begin{aligned}
& \ell y_{2}=\dot{\omega}_{2}-\ell \alpha_{1}=-\frac{y_{2}}{\tau_{2}}-\ell \alpha_{1}, \\
& d y_{2}=\ell y_{2} d t-\frac{\partial \alpha_{1}}{\partial y} g_{1}^{T}(y) d w .
\end{aligned}
$$

From (4), we obtain

$$
\begin{aligned}
\ell\left(\frac{1}{4} y_{2}^{4}\right) & =y_{2}^{3} \ell y_{2}+\frac{3}{2} y_{2}^{2}\left(\frac{\partial \alpha_{1}}{\partial y}\right)^{2}\left\|g_{1}(y)\right\|^{2} \\
& =-\frac{y_{2}^{4}}{\tau_{2}}-y_{2}^{3} \ell \alpha_{1}+\frac{3}{2} y_{2}^{2}\left(\frac{\partial \alpha_{1}}{\partial y}\right)^{2}\left\|g_{1}(y)\right\|^{2}
\end{aligned}
$$

There exist two nonnegative continuous functions $\eta_{2}\left(\bar{s}_{2}, y_{2}\right.$, $\left.\widehat{\theta}_{1}, \widehat{b}_{m}, \widehat{\bar{b}}, \xi, \bar{\lambda}_{m+2}, \varepsilon_{2}, y_{d}, \dot{y}_{d}, \ddot{y}_{d}\right)$ and $\zeta_{2}\left(\bar{s}_{2}, y_{2}, \widehat{\theta}_{1}, \widehat{b}_{m}, \widehat{\bar{b}}, \xi, \bar{\lambda}_{m+2}\right.$, $\left.\varepsilon_{2}, y_{d}, \dot{y}_{d}, \ddot{y}_{d}\right)$ such that

$$
\begin{aligned}
& \left|\ell y_{2}+\frac{y_{2}}{\tau_{2}}\right| \\
& \leq \eta_{2}\left(\bar{s}_{2}, y_{2}, \widehat{\theta}_{1}, \widehat{b}_{m}, \widehat{\bar{b}}, \xi, \bar{\lambda}_{m+2}, \varepsilon_{2}, y_{d}, \dot{y}_{d}, \ddot{y}_{d}\right), \\
& \frac{3}{2} y_{2}^{2}\left(\frac{\partial \alpha_{1}}{\partial y}\right)^{2}\left\|g_{1}(y)\right\|^{2} \\
& \leq \zeta_{2}\left(\bar{s}_{2}, y_{2}, \widehat{\theta}_{1}, \widehat{b}_{m}, \widehat{\bar{b}}, \xi, \bar{\lambda}_{m+2}, \varepsilon_{2}, y_{d}, \dot{y}_{d}, \ddot{y}_{d}\right) .
\end{aligned}
$$


From (53), we have

$$
\begin{aligned}
y_{2}^{3} \ell y_{2} \leq & -\frac{y_{2}^{4}}{\tau_{2}} \\
& +\left|y_{2}^{3}\right| \eta_{2}\left(\bar{s}_{2}, y_{2}, \widehat{\theta}_{1}, \widehat{b}_{m}, \widehat{\bar{b}}, \xi, \bar{\lambda}_{m+2}, \varepsilon_{2}, y_{d}, \dot{y}_{d}, \ddot{y}_{d}\right) \\
\leq & -\frac{y_{2}^{4}}{\tau_{2}}+\frac{3}{4} y_{2}^{4}+\frac{1}{4} \eta_{2}^{4} .
\end{aligned}
$$

From (52), (54), and (55), we obtain

$$
\ell\left(\frac{1}{4} y_{2}^{4}\right) \leq-\frac{y_{2}^{4}}{\tau_{2}}+\frac{3}{4} y_{2}^{4}+\frac{1}{4} \eta_{2}^{4}+\zeta_{2} .
$$

The infinitesimal generator of $y_{i+1}$ is

$$
\begin{aligned}
\ell y_{i+1}= & -\frac{y_{i+1}}{\tau_{i+1}}-\ell \alpha_{i} \\
y_{i+1}^{3} \ell y_{i+1}= & -\frac{y_{i+1}^{4}}{\tau_{i+1}}-y_{i+1}^{3} \ell \alpha_{i} \\
\ell\left(\frac{y_{i+1}^{4}}{4}\right)= & -\frac{y_{i+1}^{4}}{\tau_{i+1}}-y_{i+1}^{3} \ell \alpha_{i} \\
& +\frac{3}{2} y_{i+1}^{2}\left(\frac{\partial \alpha_{i}}{\partial y}\right)^{2}\left\|g_{1}(y)\right\|^{2} .
\end{aligned}
$$

There exist two nonnegative continuous functions $\eta_{i+1}\left(\bar{s}_{i+1}\right.$, $\left.\bar{y}_{i+1}, \widehat{\theta}_{1}, \widehat{b}_{m}, \widehat{\bar{b}}, \xi, \bar{\lambda}_{m+2}, \varepsilon_{2}, y_{d}, \dot{y}_{d}, \ddot{y}_{d}\right)$ and $\zeta_{i+1}\left(\bar{s}_{i+1}, \bar{y}_{i+1}, \widehat{\theta}_{1}, \widehat{b}_{m}\right.$, $\left.\widehat{\bar{b}}, \xi, \lambda_{m+2}, \varepsilon_{2}, y_{d}, \dot{y}_{d}, \ddot{y}_{d}\right)$ such that the following inequalities hold:

$$
\begin{aligned}
& \left|\ell y_{i+1}+\frac{y_{i+1}}{\tau_{i+1}}\right| \\
& \leq \eta_{i+1}\left(\bar{s}_{i+1}, \bar{y}_{i+1}, \widehat{\theta}_{1}, \widehat{b}_{m}, \widehat{\bar{b}}, \xi, \bar{\lambda}_{m+2}, \varepsilon_{2}, y_{d}, \dot{y}_{d}, \ddot{y}_{d}\right) \\
& \frac{3}{2} y_{i+1}^{2}\left(\frac{\alpha}{\partial y}\right)^{2}\left\|g_{1}(y)\right\|^{2} \\
& \leq \zeta_{i+1}\left(\bar{s}_{i+1}, \bar{y}_{i+1}, \widehat{\theta}_{1}, \widehat{b}_{m}, \widehat{\bar{b}}, \xi, \bar{\lambda}_{m+2}, \varepsilon_{2}, y_{d}, \dot{y}_{d}, \ddot{y}_{d}\right) .
\end{aligned}
$$

From (59), we obtain

$$
\begin{aligned}
& y_{i+1}^{3} \ell y_{i+1} \\
& \leq-\frac{y_{i+1}^{4}}{\tau_{i+1}} \\
& \quad+\left|y_{i+1}^{3}\right| \eta_{i+1}\left(\bar{s}_{i+1}, \bar{y}_{i+1}, \widehat{\theta}_{1}, \widehat{b}_{m}, \widehat{\bar{b}}, \xi, \bar{\lambda}_{m+2}, \varepsilon_{2}, y_{d}, \dot{y}_{d}, \ddot{y}_{d}\right) \\
& \leq-\frac{y_{i+1}^{4}}{\tau_{i+1}}+\frac{3 y_{i+1}^{4}}{4}+\frac{\eta_{i+1}^{4}}{4} .
\end{aligned}
$$

From (58), (60), and (61), we obtain

$$
\ell\left(\frac{1}{4} y_{i+1}^{4}\right) \leq-\frac{y_{i+1}^{4}}{\tau_{i+1}}+\frac{3}{4} y_{i+1}^{4}+\frac{1}{4} \eta_{i+1}^{4}+\zeta_{i+1} .
$$

The continuous function $S(\cdot)$ on the compact set $\Omega_{d} \times \Omega_{1}$ has a maximum $M(p)$, which depends on the constant $p$, and $\kappa\left(s_{1}, y_{d}, \dot{y}_{d}\right)$ on the compact set $\Omega_{d} \times \Omega_{1}$ has a maximum $N_{0}(p), \eta_{i+1}(\cdot)$ and $\zeta_{i+1}(\cdot)$ on the compact set $\Omega_{d} \times \Omega_{i+1}$ have the maximum $N_{i+1}(p)$ and $C_{i+1}(p)$ when $\xi, \bar{\lambda}_{m+2}$ are bounded.

Theorem 11. Consider the closed-loop system consisting of the plant (1) under Assumptions 1-5, the controller (46), and the adaptation laws (48) and (49). For any bounded initial conditions, there exist constants $k_{i}, \tau_{i}, h, \gamma_{1}, \gamma_{2}, \gamma_{3}, \sigma_{1}, \sigma_{2}, \sigma_{3}$ satisfying $V(0) \leq c$, such that all of the signals in the closed-loop system are bounded in probability, and $s_{1}, \ldots, s_{\rho}, y_{2}, \ldots, y_{\rho}$ are SGUUB in four-moment, $\widetilde{\theta}_{1}, \widetilde{b}_{m}, \widetilde{\bar{b}}$ are SGUUB in mean square, and $k_{i}, \tau_{i}$, and hatisfy

$$
\begin{aligned}
k_{i} & \geq \frac{3}{2} b_{\max }+\frac{9}{4}+\frac{1}{4} \alpha_{0}, \quad i=1,2, \ldots, \rho, \\
\frac{1}{\tau_{i}} & \geq \frac{1}{4} b_{\max }+1+\frac{1}{4} \alpha_{0}, \quad i=2, \ldots, \rho, \\
h & \geq \frac{9}{4}+\alpha_{0} \lambda_{\max }(P), \\
\alpha_{0} & =\min \left\{\frac{c_{3}}{2 c_{2}}, \gamma_{1} \sigma_{1}, \gamma_{2} \sigma_{2}, \gamma_{3} \sigma_{3}\right\},
\end{aligned}
$$

where $c>0$ is a positive constant; $V$ will be given later in the proof of Theorem 11.

Proof. Choose the following Lyapunov function candidate:

$$
\begin{aligned}
V= & V_{s W \varepsilon}+\sum_{i=2}^{\rho} V_{s_{i}}+\frac{1}{4} \sum_{i=2}^{\rho} y_{i}^{4}+\frac{1}{2 \gamma_{1}} \widetilde{\theta}_{1}^{T} \widetilde{\theta}_{1}+\frac{1}{2 \gamma_{2}} \widetilde{b}_{m}^{2} \\
& +\frac{1}{2 \gamma_{3}} \widetilde{\bar{b}}^{T} \widetilde{\bar{b}}
\end{aligned}
$$

For any given positive constant, if $E V \leq c$, according to Lemma 9, we obtain that $s_{1}, \ldots, s_{\rho}, y_{2}, \ldots, y_{\rho}, y, \widehat{\theta}_{1}, \widehat{b}_{m}, \widehat{\bar{b}}$ are bounded in probability. $V_{W} \leq V_{s W \varepsilon}=V_{s 1}+V_{W}+V_{\varepsilon} \leq$ $V \leq c$, and, from Assumption 2, we obtain that $\left(c_{1} / \lambda_{0}\right)\|z\|^{4} \leq$ $\left(1 / \lambda_{0}\right) W \leq c$; that is, $\|z\|^{4} \leq \lambda_{0} c / c_{1}$, so $z$ is bounded in probability.

Furthermore, (64) is rewritten as $V=\left(1 / \lambda_{0}\right) W+$ $(1 / 2) V_{\rho}-\left(c_{3} / 2 \lambda_{0}\right)\|z\|^{4}$; then $V_{\rho}=2 V-\left(2 / \lambda_{0}\right) W+$ $\left(c_{3} / \lambda_{0}\right)\left\|z^{4}\right\| \leq\left(2+c_{3} / c_{1}\right) c$, and choosing $p=\left(2+c_{3} / c_{1}\right) c$, we get $V_{\rho} \leq p$.

From (14) and (49), we have that $\xi, \bar{\omega}, \alpha_{1}$ are also bounded in probability. It yields that $v_{m-1,2}, \ldots, v_{0,2}$ are all bounded in probability. Noting $v_{m, 2}=s_{2}+y_{2}+\alpha_{1}$, we obtain that $v_{m, 2}$ is bounded in probability. From (14), we have that $\dot{v}_{0,1}=$ $-l_{1} v_{0,1}+v_{0,2}$ and $\dot{v}_{m, 1}=-l_{1} v_{m, 1}+v_{m, 2}$. Thus we obtain that $v_{0,1}, v_{m, 1}$ are also bounded. Furthermore, from (42), we have 
that $\alpha_{i}(i=2, \ldots, \rho-1)$ are bounded. According to (16) and (17), we obtain

$$
\left[\begin{array}{c}
v_{0,1} \\
v_{0,2} \\
v_{1,2} \\
\vdots \\
v_{m-1,2}
\end{array}\right]=\left[\begin{array}{ccccc}
1 & 0 & 0 & \cdots & 0 \\
0 & 1 & 0 & \cdots & 0 \\
* & * & 1 & \cdots & 0 \\
\vdots & & & \ddots & \vdots \\
* & * & \cdots & * & 1
\end{array}\right]\left[\begin{array}{c}
\lambda_{1} \\
\lambda_{2} \\
\lambda_{3} \\
\vdots \\
\lambda_{m+1}
\end{array}\right] .
$$

Since $v_{0,1}, v_{0,2}, v_{1,2}, \ldots, v_{m-1,2}$ are bounded in probability, we have that $\lambda_{1}, \ldots, \lambda_{m+1}$ are all bounded in probability. From (17), we get that $\lambda_{m+2}$ is also bounded. In view of (40), (44), (47)-(49), and (62), using Young's inequality, we obtain

$$
\begin{aligned}
\ell V \leq & -\left(h-\frac{9}{4}\right) \varepsilon^{T} \varepsilon-\sum_{i=1}^{\rho}\left(k_{i}-\frac{3}{2} b_{\max }-\frac{9}{4}\right) s_{i}^{4} \\
& -\sum_{i=2}^{\rho}\left(\frac{1}{\tau_{i}}-\frac{1}{4} b_{\max }-1\right) y_{i}^{4}-\frac{c_{3}}{2 \lambda_{0}}\|z\|^{4} \\
& -\frac{\sigma_{1}\left\|\widetilde{\theta}_{1}\right\|^{2}}{2}-\frac{\sigma_{2} \widetilde{b}_{m}^{2}}{2}-\frac{\sigma_{3}\|\widetilde{b}\|^{2}}{2}+Q(y) \\
& +\frac{1}{4} N_{0}^{4}(p)+\frac{1}{4} M^{4}(p)+\frac{1}{4} \sum_{i=2}^{\rho} N_{i}^{4}(p) \\
& +\sum_{i=2}^{\rho} C_{i}(p)+\frac{\sigma_{1}\left\|\theta_{1}\right\|^{2}}{2}+\frac{\sigma_{2} b_{m}^{2}}{2}+\frac{\sigma_{3}\|\bar{b}\|^{2}}{2}+C_{0} .
\end{aligned}
$$

Substituting (63) into (66), we obtain

$$
\ell V \leq-\alpha_{0} V+\mu_{1}+\mu_{0}
$$

where $\mu_{1}=(1 / 4) N_{0}^{4}(p)+(1 / 4) M^{4}(p)+(1 / 4) \sum_{i=2}^{\rho} N_{i}^{4}(p)+$ $\sum_{i=2}^{\rho} C_{i}(p)+\sigma_{1}\left\|\theta_{1}\right\|^{2} / 2+\sigma_{2} b_{m}^{2} / 2+\sigma_{3}\|\bar{b}\|^{2} / 2+C_{0}$. Since $|Q(y)|$ is a nonnegative continuous function, let $|Q(y)| \leq \mu_{0}$, where $\mu_{0}>0$.

If $\alpha_{0} \geq\left(\mu_{1}+\mu_{0}\right) / c$, and $E V=c$, then we have $d E V / d t \leq 0$. Thus, if $E V(0) \leq c$, then $E V(t) \leq c, \forall t>0$; that is,

$$
\begin{aligned}
0 & \leq E V(t) \leq \frac{\mu_{1}+\mu_{0}}{\alpha_{0}}+\left[V(0)-\frac{\mu_{1}+\mu_{0}}{\alpha_{0}}\right] e^{-\alpha_{0} t} \\
& \leq V(0) .
\end{aligned}
$$

Similar to the discussion of Theorem 11 in [32], it is easy to know that the conclusion is true.

Remark 12. This paper differs from [32] in the following several aspects. (1) Unmodeled dynamics is dealt with by introducing a novel description based on Lyapunov function in this paper while the dynamic signal was handled with the help of a dynamic signal in [32]. (2) The unknown nonlinear system functions are handled together with some functions produced in stability analysis, but they were directly approximated before constructing the observer in [32]. Therefore, this brings out a good result that the filter order is reduced. (3)
The neural networks weight vector used to approximate the black box function at the first design step is adjusted online in this paper such that much more information of weight vector can be used in adaptive law, whereas only the norm of weight vector acts as adaptive tuning parameter in [32]. (4) Utilizing bounded input bounded output stability and linear equations (65), the stability of the closed-loop system is proved in this paper, which avoids using the transfer function to make stability analysis in [32], which is questionable in probability sense.

Remark 13. The design parameters $k_{i}, \tau_{i}$ and $\alpha_{0}$ determined by (63) in Theorem 11 are only a sufficient condition. They provide a guideline for the designers. From (63), some suggestions are given for the choice of some key design parameters for any given positive constants $B_{0}$ and $c$.

(i) Increasing $\gamma_{1}, \gamma_{2}, \gamma_{3}$ helps to increase $\alpha_{0}$, subsequently reduces $\mu_{1} / \alpha_{0}$.

(ii) Decreasing $\sigma_{1}, \sigma_{2}, \sigma_{3}$ helps to reduce $\mu_{1}$ and reduces $\mu_{1} / \alpha_{0}$.

(iii) Increasing $k_{1}, \ldots, k_{\rho}$ helps to increase $\alpha_{0}$ and reduces $\mu_{1} / \alpha_{0}$.

In practical applications, to obtain good tracking performance, some experiments need to be done before the valid parameters are given.

\section{Simulation Results}

To demonstrate the effectiveness of the proposed approach, two numerical examples are given.

Example 1. Consider the following third-order stochastic nonlinear system with unmodeled dynamics:

$$
\begin{gathered}
\dot{z}=q(z, y) \\
d x_{1}=\left(x_{2}+\frac{y-y^{3}}{1+y^{2}}+0.5 z\right) d t+y \sin \left(y^{3}\right) d w \\
d x_{2}=\left(x_{3}+\frac{y-y^{3}}{1+y^{2}}+0.5 z+0.2\left(35+y^{2}\right) u\right) d t \\
+x_{1} \sin \left(y^{3}\right) d w
\end{gathered}
$$

$d x_{3}$

$$
\begin{aligned}
= & \left(y^{2} \tanh (y)-\left(y^{2}+2 y\right) \sin y+0.2\left(35+y^{2}\right) u+y z\right) d t \\
& +0.5 y^{2} d w,
\end{aligned}
$$

$$
y=x_{1}
$$

where $q(z, y)=-2 z+y \sin t+0.5, m=1, \rho=2$. The desired tracking trajectory is taken as $y_{d}=0.5 \sin (0.5 t)$. Select $W(z, t)=(1 / 4) z^{4}, c_{1}=1 / 8, c_{2}=1, c_{3}=2, c_{4}=1$, 
$c_{5}=0.5$; then $(\partial W / \partial t)(z, t)+(\partial W / \partial z)(z, t)(q(z, 0, t)-$ $q(0,0, t))=-2 z^{4},|(\partial W / \partial z)(z, t)|=|z|^{3},|q(0,0, t)|=0.5$; $\psi_{0}(|y|)=|y|,|q(z, y, t)-q(z, 0, t)|=|y \sin t| \leq \psi_{0}(|y|)$, and it satisfies the conditions of Assumptions 2 and 3.

The filters are designed as follows:

$$
\begin{aligned}
& \dot{\xi}_{1}=-l_{1} \xi_{1}+\xi_{2}+l_{1} y, \\
& \dot{\xi}_{2}=-l_{2} \xi_{1}+\xi_{3}+l_{2} y, \\
& \dot{\xi}_{3}=-l_{3} \xi_{1}+l_{3} y, \\
& \dot{\lambda}_{1}=-l_{1} \lambda_{1}+\lambda_{2}, \\
& \dot{\lambda}_{2}=-l_{2} \lambda_{1}+\lambda_{3}, \\
& \dot{\lambda}_{3}=-l_{3} \lambda_{1}+\sigma(y) u .
\end{aligned}
$$

The adaptation laws are employed as follows:

$$
\begin{aligned}
& \dot{\hat{\theta}}_{1}=\gamma_{1}\left(s_{1}^{3} \psi_{1}(X)-\sigma_{1} \widehat{\theta}_{1}\right), \\
& \dot{\vec{b}}_{1}=\gamma_{2}\left(s_{1}^{3} \alpha_{1}-\sigma_{2} \widehat{b}_{1}\right), \\
& \dot{\vec{b}}_{0}=\gamma_{3}\left(s_{1}^{3} v_{0,2}-\sigma_{3} \widehat{b}_{0}\right),
\end{aligned}
$$

where $X=\left[s_{1}, y_{d}, \dot{y}_{d}\right]^{T}$.

The virtual control law $\alpha_{1}$ is chosen as follows:

$$
\alpha_{1}=\frac{\widehat{b}_{1}}{\widehat{b}_{1}^{2}+\beta}\left(-k_{1} s_{1}-\xi_{2}-v_{0,2} \widehat{b}_{0}-s_{1}^{3} \widehat{\theta}_{1}^{T} \psi_{1}(X)\right) .
$$

The control law is employed as follows:

$$
u=\frac{\left(-k_{2} s_{2}+l_{2} v_{0,1}+\dot{\omega}_{2}\right)}{\sigma(y)}
$$

where $v_{0,1}=\lambda_{1}, v_{0,2}=\lambda_{2}, \sigma(y)=35+y^{2}$.

In the simulation, $s_{1}=y-y_{d}, s_{2}=v_{0,2}-\omega_{2}, l_{1}=6$, $l_{2}=11, l_{3}=6, k_{1}=40, k_{2}=50, \beta=0.02, \omega_{2}(0)=0.1$, $\tau_{2}=0.01, \gamma_{1}=\gamma_{2}=\gamma_{3}=2, \sigma_{1}=\sigma_{2}=\sigma_{3}=0.05, x(0)=$ $[0.2,0,0]^{T}, \xi(0)=[0,0,0]^{T}, \lambda(0)=[0,0,0]^{T}, \widehat{b}_{0}(0)=\widehat{b}_{1}(0)=$ $1, \widehat{\theta}_{1}(0)=[0.1]_{1 \times 10}^{T}, M_{1}=10$. Simulation results are shown in Figures 1, 2, and 3. From Figure 1, it can be seen that fairly good tracking performance is obtained.

Remark 14. According to (69), we know that $b_{1}=0.2$ and $b_{\max }=1$. From the above selected design parameters and (63), it is easy to see that $c_{3} /\left(2 c_{2}\right)=1, \alpha_{0}=0.1$. The constant $h$ is only used to analyze the stability in the closed-loop system. Therefore, (63) is true for the above selected design parameters $\gamma_{1}, \gamma_{2}, \gamma_{3}, \sigma_{1}, \sigma_{2}, \sigma_{3}, k_{1}, k_{2}, \tau_{2}$.

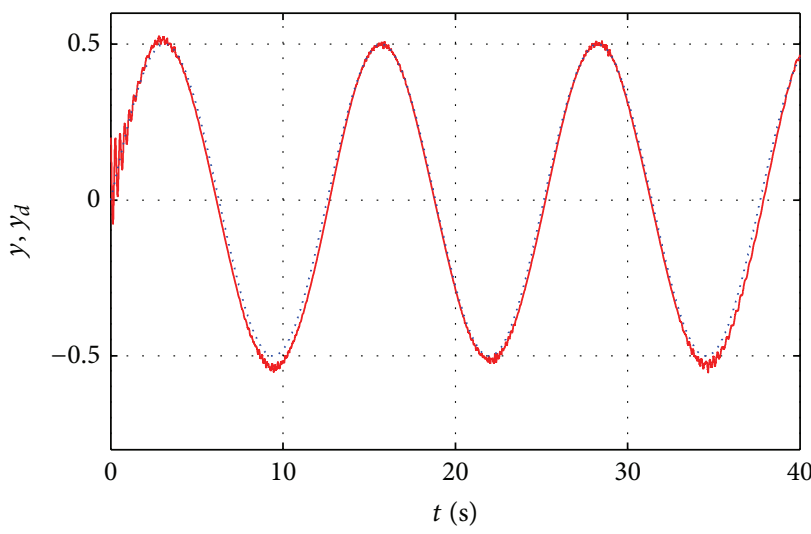

FIGURE 1: Output $y$ (solid line) and desired trajectory $y_{d}$ (dotted line).

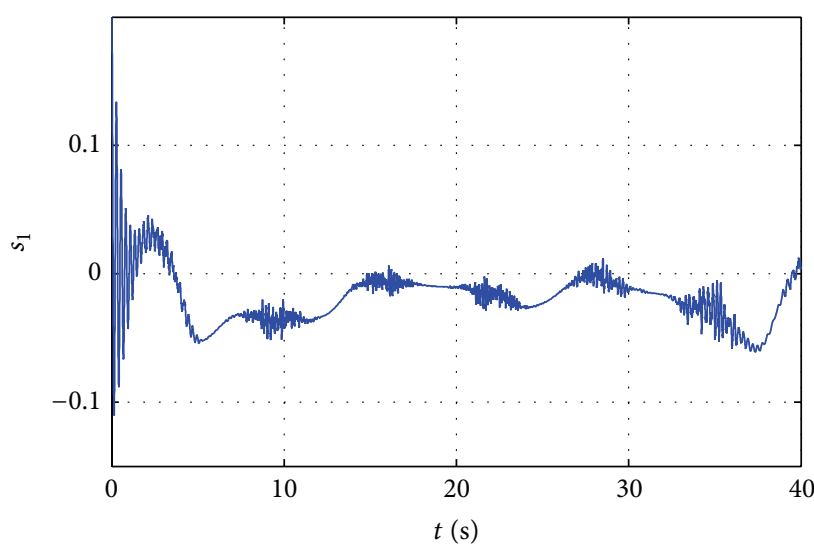

Figure 2: Tracking error $s_{1}$.

Example 2. To compare the simulation results with [32], consider the following same stochastic nonlinear system with unmodeled dynamics in [32]:

$$
\dot{z}=q(z, y),
$$

$$
\begin{gathered}
d x_{1}=\left(x_{2}+\frac{x_{1}-x_{1}^{3}}{1+x_{1}^{2}}+0.5 z\right) d t+x_{1} \sin \left(x_{1}^{3}\right) d w \\
d x_{2}=\left(x_{1}^{2} \tanh \left(x_{1}\right)-\left(x_{1}^{2}+2 x_{1}\right) \sin x_{1}\right. \\
\left.+0.2\left(0.5+x_{1}^{2}\right) u+x_{1} z\right) d t \\
+0.5 x_{1}^{2} d w \\
y=x_{1},
\end{gathered}
$$




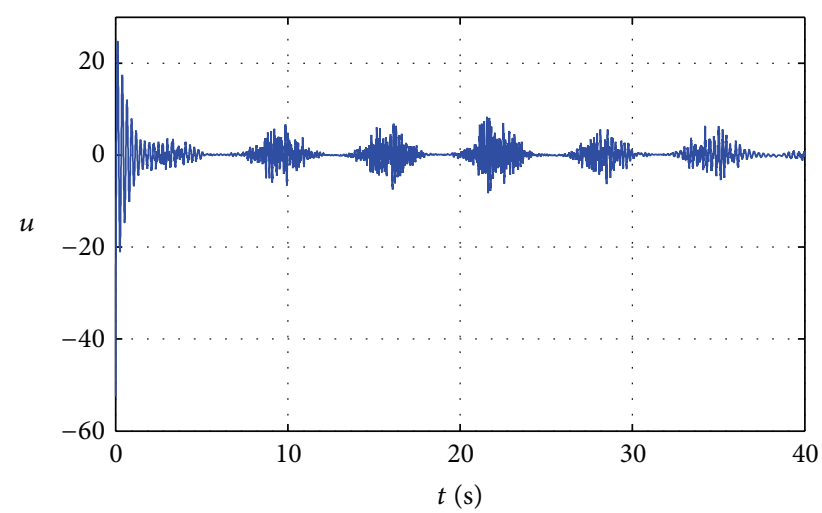

FIGURE 3: Control signal $u$.

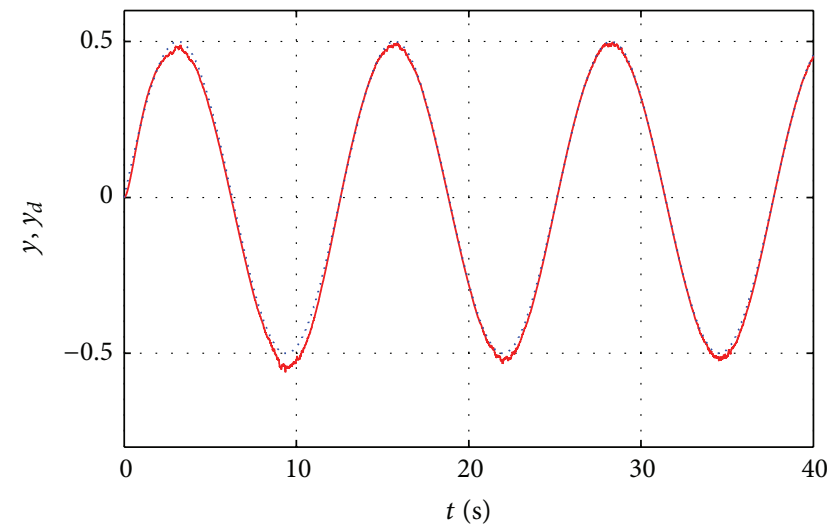

Figure 4: Output $y$ (solid line) and desired trajectory $y_{d}$ (dotted line).

where $q(z, y)=-2 z+y^{2}, m=0, \rho=2$. The desired tracking trajectory is taken as $y_{d}=0.5 \sin (0.5 t)$. The filters are designed as follows:

$$
\begin{aligned}
& \dot{\xi}_{1}=-l_{1} \xi_{1}+\xi_{2}+l_{1} y, \\
& \dot{\xi}_{2}=-l_{2} \xi_{1}+l_{2} y, \\
& \dot{\lambda}_{1}=-l_{1} \lambda_{1}+\lambda_{2}, \\
& \dot{\lambda}_{2}=-l_{2} \lambda_{1}+\sigma(y) u .
\end{aligned}
$$

The adaptation laws are employed as follows:

$$
\begin{aligned}
& \dot{\hat{\theta}}_{1}=\gamma_{1}\left(s_{1}^{3} \psi_{1}(X)-\sigma_{1} \widehat{\theta}_{1}\right), \\
& \dot{\widehat{b}}_{0}=\gamma_{3}\left(s_{1}^{3} v_{0,2}-\sigma_{3} \widehat{b}_{0}\right),
\end{aligned}
$$

where $X=\left[s_{1}, y_{d}, \dot{y}_{d}\right]^{T}$.

The virtual control law $\alpha_{1}$ is chosen as follows:

$$
\alpha_{1}=\frac{\widehat{b}_{0}}{\widehat{b}_{0}^{2}+\beta}\left(-k_{1} s_{1}-\xi_{2}-\lambda_{2} \widehat{b}_{0}-s_{1}^{3} \widehat{\theta}_{1}^{T} \psi_{1}(X)\right) \text {. }
$$

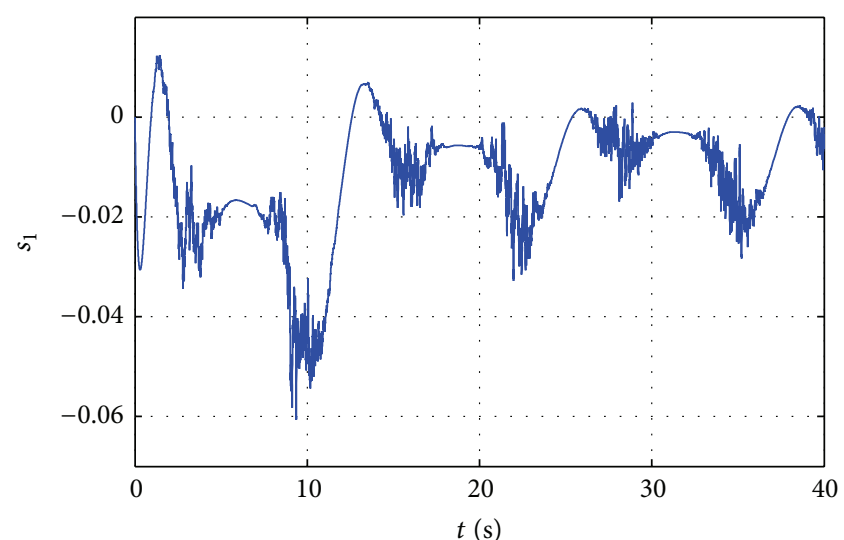

FIGURE 5: Tracking error $s_{1}$.

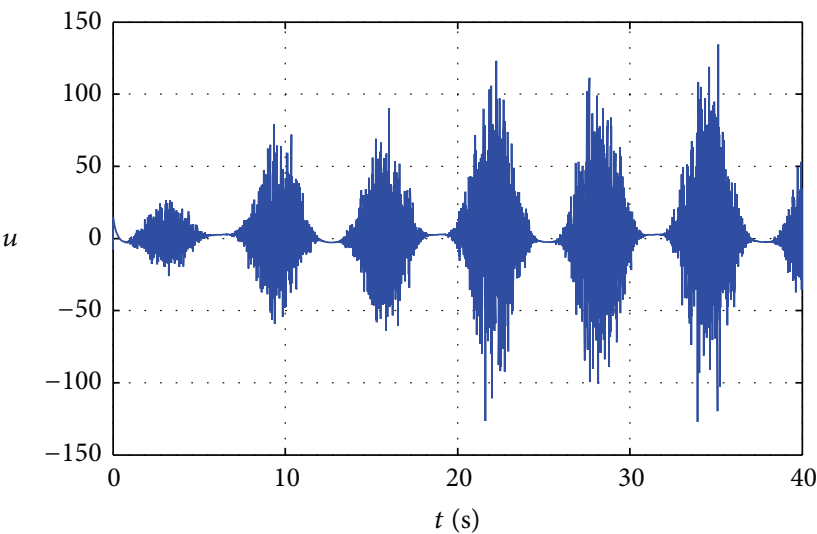

FIgURE 6: Control signal $u$.

The control law is employed as follows:

$$
u=\frac{\left(-k_{2} s_{2}+l_{2} v_{0,1}+\dot{\omega}_{2}\right)}{\sigma(y)}
$$

where $v_{0,1}=\lambda_{1}, \sigma(y)=0.5+y^{2}$.

In the simulation, $s_{1}=y-y_{d}, s_{2}=v_{0,2}-\omega_{2}, l_{1}=5, l_{2}=6$, $k_{1}=60, k_{2}=60, \beta=0.02, \omega_{2}(0)=0.1, \tau_{2}=0.01, \gamma_{1}=\gamma_{3}=$ $1.5, \sigma_{1}=\sigma_{3}=0.05, x(0)=[0,0]^{T}, z(0)=0, \xi(0)=[0,0]^{T}$, $\lambda(0)=[0,0]^{T}, \widehat{b}_{0}(0)=1, \widehat{\theta}_{1}(0)=[0.1,0.1,0.1,0.1,0.1]^{T}$, $M_{1}=5$. Simulation results are shown in Figures $4-6$. If the proposed approach in [32] is utilized, and the design parameters of the adaptive controller are taken, the same values as in [32], the corresponding simulation results are as shown in Figures 7-9.

From Figures 4, 5, 7, and 8, it can be seen that better tracking performance can be obtained than [32]. However, 42 equations need to be solved online using the method in [32] while only 14 equations need to be solved online using the approach in this paper. Moreover, we know that increasing $k_{1}, k_{2}$ helps to improve the tracking precision. 


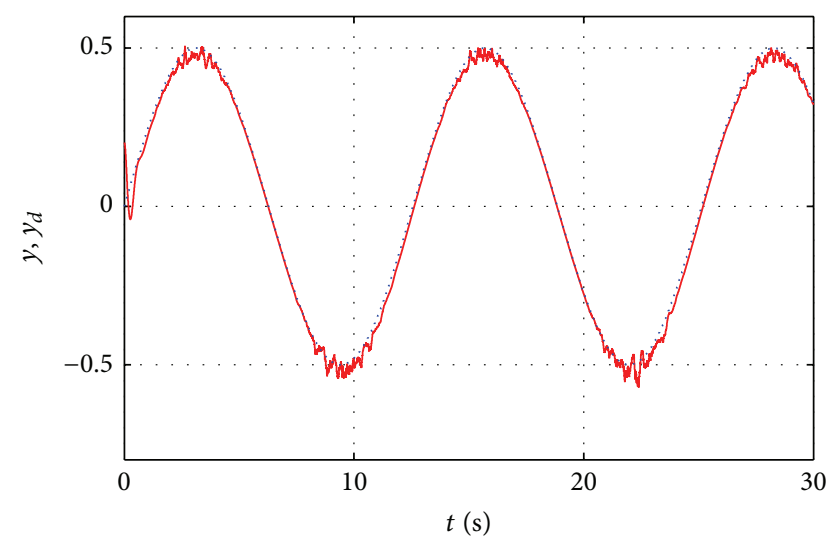

FIGURE 7: Output $y$ (solid line) and desired trajectory $y_{d}$ (dotted line).

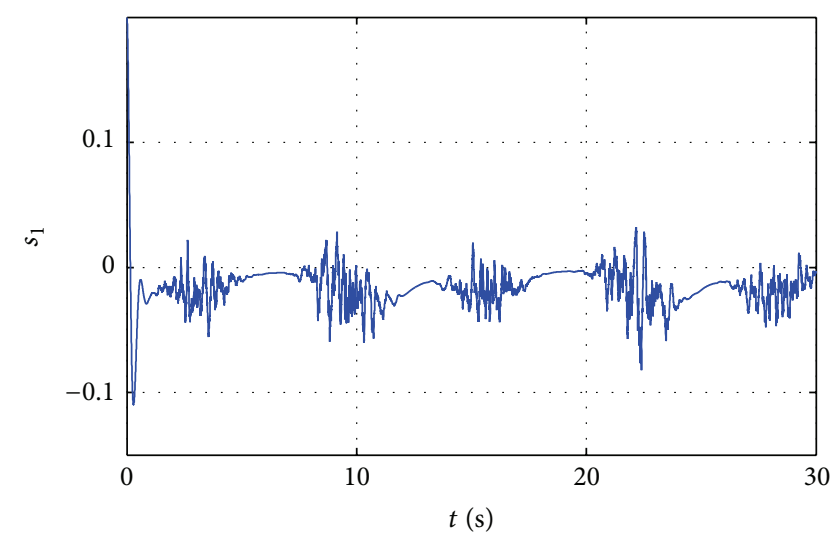

FIgURE 8: Tracking error $s_{1}$.

\section{Conclusions}

Using K-filters and dynamic surface control, an adaptive output feedback neural control scheme has been proposed for a class of stochastic nonlinear systems with unmodeled dynamics. Unmodeled dynamics has been dealt with by introducing the novel description based on Lyapunov function. The unknown nonlinear system functions are handled together with some functions resulting from stability analysis, and the filter order is reduced. The neural network weight vector is adjusted online. Therefore, the more information included in radial basis function can be fully made use of. Using Chebyshev's inequality and Itô formula, the designed controller can guarantee that all the signals in the closedloop system are bounded in probability and the error signals are semiglobally uniformly ultimately bounded in the sense of four-moment or mean square. Simulation results illustrate the effectiveness of the proposed approach.

\section{Conflict of Interests}

The authors declare that there is no conflict of interests regarding the publication of this paper.

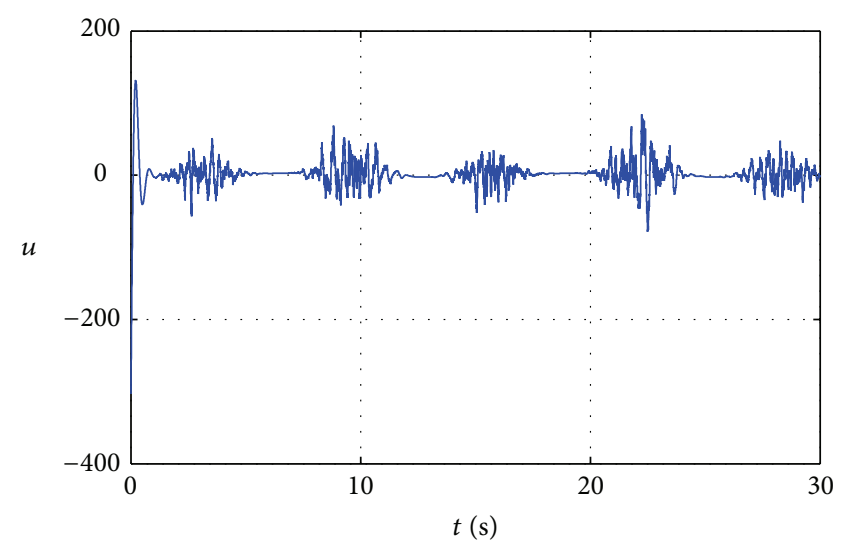

FIGURE 9: Control signal $u$.

\section{Acknowledgments}

This work was partially supported by the National Natural Science Foundation of China (61174046, 61473249, and 61473250).

\section{References}

[1] I. Kanellakopoulos, P. V. Kokotovic, and A. S. Morse, "Systematic design of adaptive controllers for feedback linearizable systems," IEEE Transactions on Automatic Control, vol. 36, no. 11, pp. 1241-1253, 1991.

[2] D. Swaroop, J. K. Hedrick, P. P. Yip, and J. C. Gerdes, "Dynamic surface control for a class of nonlinear systems," IEEE Transactions on Automatic Control, vol. 45, no. 10, pp. 1893-1899, 2000.

[3] Z. Jiang and L. Praly, "Design of robust adaptive controllers for nonlinear systems with dynamic uncertainties," Automatica, vol. 34, no. 7, pp. 825-840, 1998.

[4] Z. P. Jiang and D. J. Hill, "A robust adaptive backstepping scheme for nonlinear systems with unmodeled dynamics," IEEE Transactions on Automatic Control, vol. 44, no. 9, pp. 1705-1711, 1999.

[5] D. Wang and J. Huang, "Neural network-based adaptive dynamic surface control for a class of uncertain nonlinear systems in strict-feedback form," IEEE Transactions on Neural Networks, vol. 16, no. 1, pp. 195-202, 2005.

[6] T. S. Li, D. Wang, G. Feng, and S. C. Tong, "A DSC approach to robust adaptive nn tracking control for strict-feedback nonlinear systems," IEEE Transactions on Systems, Man, and Cybernetics B: Cybernetics, vol. 40, no. 3, pp. 915-927, 2010.

[7] T. P. Zhang and S. S. Ge, "Adaptive dynamic surface control of nonlinear systems with unknown dead zone in pure feedback form," Automatica, vol. 44, no. 7, pp. 1895-1903, 2008.

[8] T. P. Zhang, Q. Zhu, and Y. Q. Yang, "Adaptive neural control of non-affine pure-feedback non-linear systems with input nonlinearity and perturbed uncertainties," International Journal of Systems Science, vol. 43, no. 4, pp. 691-706, 2012.

[9] T. Zhang, X. Shi, Q. Zhu, and Y. Yang, "Adaptive neural tracking control of pure-feedback nonlinear systems with unknown gain signs and unmodeled dynamics," Neurocomputing, vol. 121, pp. 290-297, 2013.

[10] X. Y. Zhang and Y. Lin, "Adaptive tracking control for a class of pure-feedback non-linear systems including actuator 
hysteresis and dynamic uncertainties," IET Control Theory and Applications, vol. 5, no. 16, pp. 1868-1880, 2011.

[11] T. Zhang and S. S. Ge, "Adaptive neural network tracking control of MIMO nonlinear systems with unknown dead zones and control directions," IEEE Transactions on Neural Networks, vol. 20, no. 3, pp. 483-497, 2009.

[12] S. J. Yoo and J. B. Park, "Neural-network-based decentralized adaptive control for a class of large-scale nonlinear systems with unknown time-varying delays," IEEE Transactions on Systems, Man, and Cybernetics B: Cybernetics, vol. 39, no. 5, pp.1316-1323, 2009.

[13] S. J. Yoo, "Decentralised adaptive control of a class of interconnected non-linear systems with unknown time delays and deadzone inputs," IET Control Theory \& Applications, vol. 4, no. 11, pp. 2639-2650, 2010.

[14] M. Krstic, I. Kanellakopoulos, and P. V. Kokotocic, Nonlinear and Adaptive Control Design, Wiley-Interscience, New York, NY, USA, 1995.

[15] Y. J. Liu and W. Wang, "Adaptive output feedback control of uncertain nonlinear systems based on dynamic surface control technique," International Journal of Robust and Nonlinear Control, vol. 22, no. 9, pp. 945-958, 2012.

[16] S. Tong, C. Liu, and Y. Li, "Adaptive fuzzy backstepping output feedback control for strict feedback nonlinear systems with unknown sign of high-frequency gain," Neurocomputing, vol. 77, no. 1, pp. 58-70, 2012.

[17] Y. M. Li, S. C. Tong, Y. J. Liu, and T. S. Li, "Adaptive fuzz y robust otput feedback control of nonlinear systems with unknown dead-zones based on a small-gain approach," IEEE Transactions on Fuzzy Systems, vol. 22, no. 1, pp. 164-176, 2014.

[18] C. Wang and Y. Lin, "Decentralised adaptive dynamic surface control for a class of interconnected non-linear systems," IET Control Theory \& Applications, vol. 6, no. 9, pp. 1172-1181, 2012.

[19] H. Deng and M. Krstić, "Stochastic nonlinear stabilization-I: a backstepping design," Systems and Control Letters, vol. 32, no. 3, pp. 143-150, 1997.

[20] H. Deng and M. Krstić, "Output-feedback stochastic nonlinear stabilization," IEEE Transactions on Automatic Control, vol. 44, no. 2, pp. 328-333, 1999.

[21] H. Deng, M. Krstić, and R. J. Williams, "Stabilization of stochastic nonlinear systems driven by noise of unknown covariance," IEEE Transactions on Automatic Control, vol. 46, no. 8, pp. 12371253, 2001.

[22] H. Fan and W. Xie, "Adaptive state feedback control for a class of stochastic nonlinear systems with unknown backlashlike hysteresis," in Proceedings of the 2nd IEEE Conference on Industrial Electronics and Applications (ICIEA '07), pp. 27972801, May 2007.

[23] H. Fan, L. Han, C. Wen, and L. Xu, "Decentralized adaptive output-feedback controller design for stochastic nonlinear interconnected systems," Automatica, vol. 48, no. 11, pp. 28662873, 2012.

[24] T. Wang, S. Tong, and Y. Li, "Robust adaptive fuzzy control for a class of stochastic nonlinear systems with dynamical uncertainties," Journal of the Franklin Institute, vol. 349, no. 10, pp. 3121-3141, 2012.

[25] T. Wang, S. C. Tong, and Y. M. Li, "Robust adaptive fuzzy output feedback control for stochastic nonlinear systems with unknown control direction," Neurocomputing, vol. 106, pp. 3141, 2013.
[26] Z. J. Wu, X. J. Xie, and S. Y. Zhang, "Adaptive backstepping controller design using stochastic small-gain theorem," Automatica, vol. 43, no. 4, pp. 608-620, 2007.

[27] X. Yu and X. Xie, "Output feedback regulation of stochastic nonlinear systems with stochastic iISS inverse dynamics," IEEE Transactions on Automatic Control, vol. 55, no. 2, pp. 304-320, 2010.

[28] S. Liu and J. Zhang, "Output-feedback control of a class of stochastic nonlinear systems with linearly bounded unmeasurable states," International Journal of Robust and Nonlinear Control, vol. 18, no. 6, pp. 665-687, 2008.

[29] W. Li, Y. Jing, and S. Zhang, "Output-feedback stabilization for stochastic nonlinear systems whose linearizations are not stabilizable," Automatica, vol. 46, no. 4, pp. 752-760, 2010.

[30] X. Yu, X. J. Xie, and N. Duan, "Small-gain control method for stochastic nonlinear systems with stochastic iISS inverse dynamics," Automatica, vol. 46, no. 11, pp. 1790-1798, 2010.

[31] C. Zhao and X. Xie, "Output feedback stabilization using small-gain method and reduced-order observer for stochastic nonlinear systems," IEEE Transactions on Automatic Control, vol. 58, no. 2, pp. 523-528, 2013.

[32] T. P. Zhang and X. N. Xia, "Adaptive output feedback tracking control of stochastic nonlinear systems with dynamic uncertainties,' International Journal of Robust and Nonlinear Control, 2014.

[33] H. T. Gao, T. P. Zhang, and X. N. Xia, "Adaptive neural control of stochastic nonlinear systems with unmodeled dynamics and time-varying state delays," Journal of the Franklin Institute, vol. 351, no. 6, pp. 3182-3199, 2014.

[34] R. Z. Khasminskii, Stochastic Stability of Differential Equations, Kluwer Academic, Norwell, Mass, USA, 1980. 


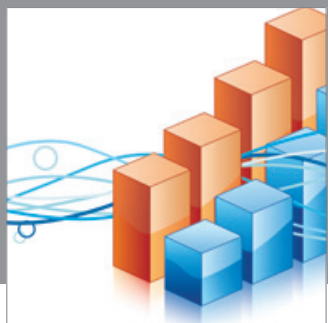

Advances in

Operations Research

mansans

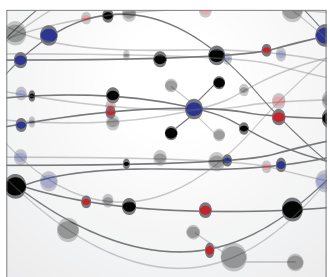

The Scientific World Journal
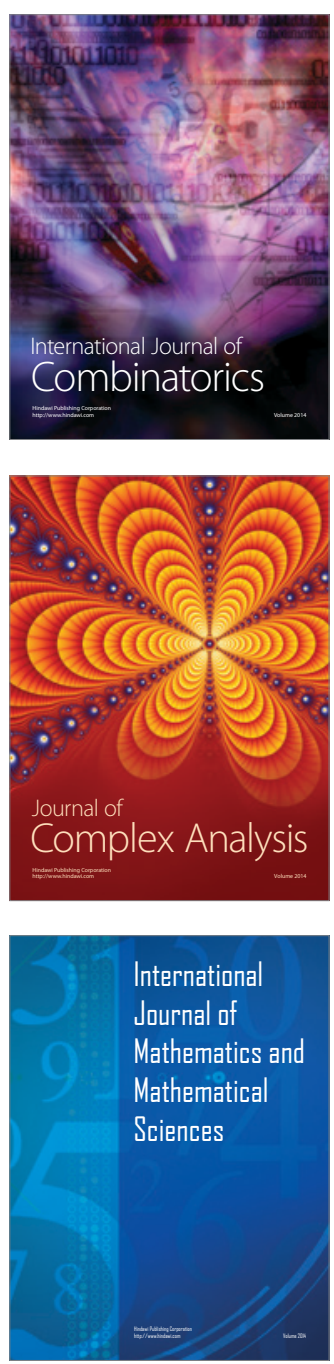
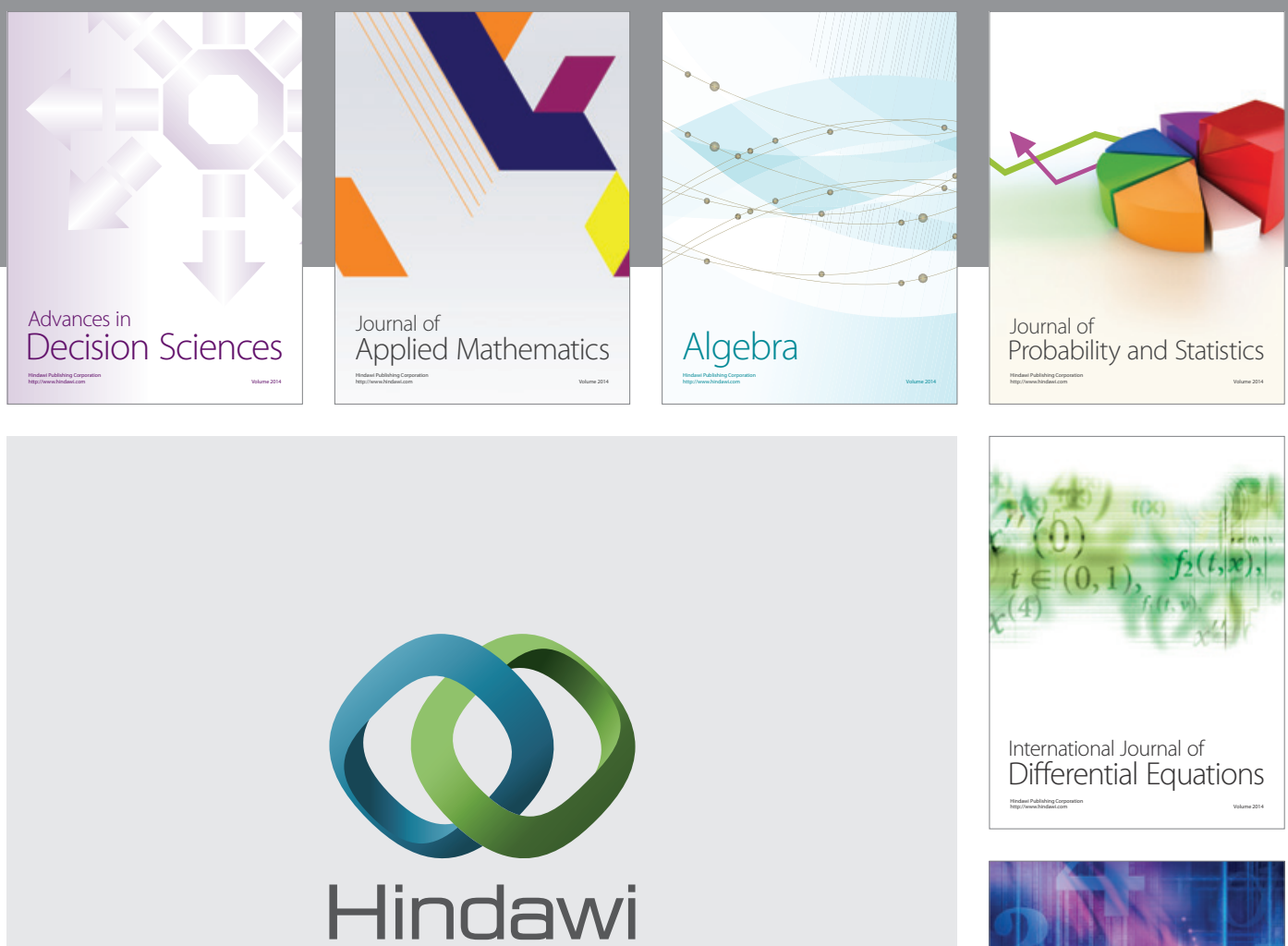

Submit your manuscripts at http://www.hindawi.com
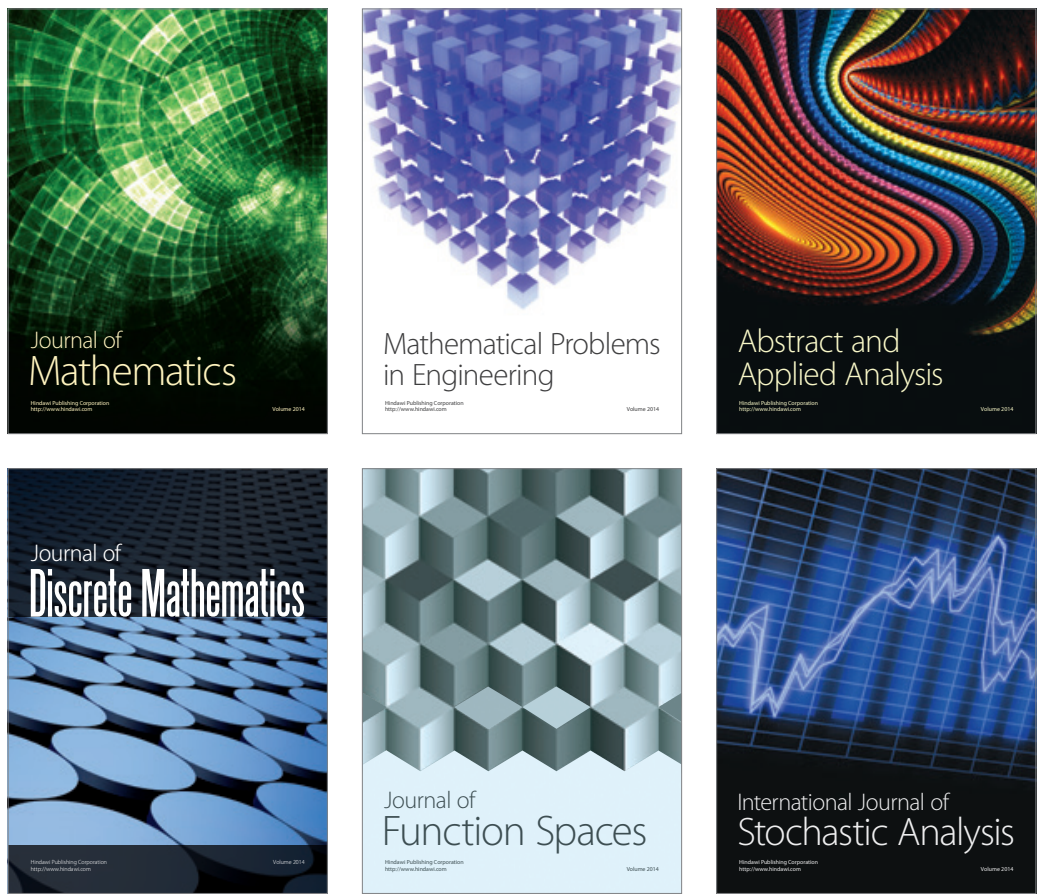

Journal of

Function Spaces

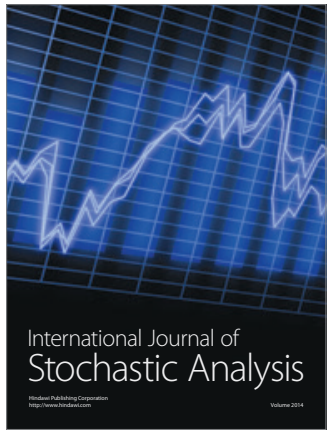

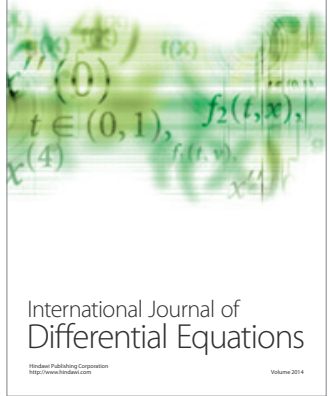
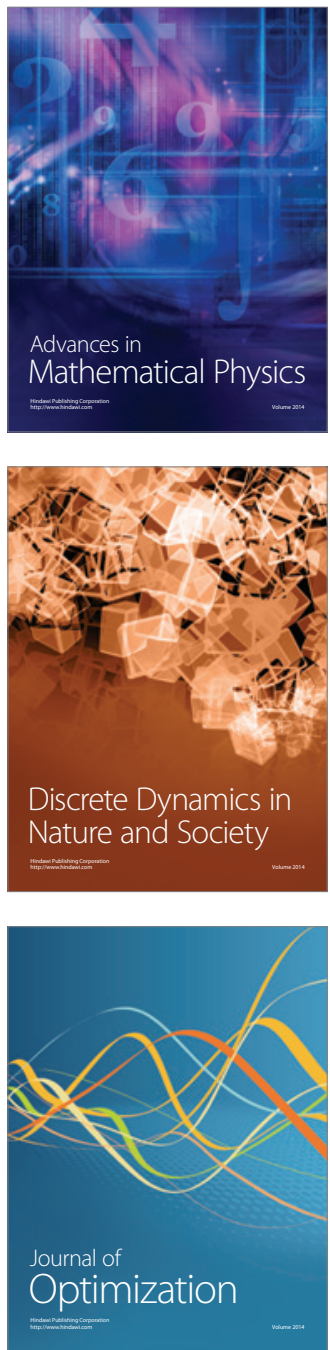